\title{
FADE: Secure Overlay Cloud Storage with File Assured Deletion
}

\author{
Yang Tang ${ }^{\dagger}$, Patrick P. C. Lee ${ }^{\dagger}$, John C. S. Lui ${ }^{\dagger}$, and Radia Perlman ${ }^{\ddagger}$ \\ ${ }^{\dagger}$ The Chinese University of Hong Kong ${ }^{\ddagger}$ Intel Labs \\ \{tangyang, pclee, cslui\}@cse.cuhk.edu.hk, radiaperlman@gmail.com
}

\begin{abstract}
While we can now outsource data backup to third-party cloud storage services so as to reduce data management costs, security concerns arise in terms of ensuring the privacy and integrity of outsourced data. We design FADE, a practical, implementable, and readily deployable cloud storage system that focuses on protecting deleted data with policy-based file assured deletion. FADE is built upon standard cryptographic techniques, such that it encrypts outsourced data files to guarantee their privacy and integrity, and most importantly, assuredly deletes files to make them unrecoverable to anyone (including those who manage the cloud storage) upon revocations of file access policies. In particular, the design of FADE is geared toward the objective that it acts as an overlay system that works seamlessly atop today's cloud storage services. To demonstrate this objective, we implement a working prototype of FADE atop Amazon S3, one of today's cloud storage services, and empirically show that FADE provides policy-based file assured deletion with a minimal trade-off of performance overhead. Our work provides insights of how to incorporate value-added security features into current data outsourcing applications.
\end{abstract}

Key words: Policy-based file assured deletion, cloud storage, prototype implementation

\section{Introduction}

Cloud storage (e.g., Amazon S3 [2], MyAsiaCloud [11]) offers an abstraction of infinite storage space for clients to host data, in a pay-as-you-go manner [3]. For example, SmugMug [19], a photo sharing website, chose to host terabytes of photos on Amazon S3 in 2006 and saved about 500K US dollars on storage devices [1]. Thus, instead of self-maintaining data centers, enterprises can now outsource the storage of a bulk amount of digitized content to those third-party cloud storage providers so as to save the financial overhead in data management. Apart from enterprises, individuals can also benefit from cloud storage as a result of the advent of mobile devices (e.g., smartphones, laptops). Given that mobile devices have limited storage space in general, individuals can move audio/video files to the cloud and make effective use of space in their mobile devices.

However, privacy and integrity concerns become relevant as we now count on third parities to host possibly sensitive data. To protect outsourced data, 
a straightforward approach is to apply cryptographic encryption onto sensitive data with a set of encryption keys, yet maintaining and protecting such encryption keys will create another security issue. One specific issue is that upon requests of deletion of files, cloud storage providers may not completely remove all file copies (e.g., cloud storage providers may make multiple file backup copies and distribute them over the cloud for reliability, and clients do not know the number or even the existence of these backup copies), and eventually have the data disclosed if the encryption keys are unexpectedly obtained, either by accidents or by malicious attacks. Therefore, we seek to achieve a major security goal called file assured deletion, meaning that files are reliably deleted and remain permanently unrecoverable and inaccessible by any party.

The security concerns motivate us, as cloud clients, to develop a secure cloud storage system that provides file assured deletion. However, a key challenge of building such a system is that cloud storage infrastructures are externally owned and managed by third-party cloud providers, and hence the system should never assume any structural changes (in protocol or hardware levels) in cloud infrastructures. Thus, it is important to design a secure overlay cloud storage system that can work seamlessly atop existing cloud storage services.

In this paper, we present FADE, a secure overlay cloud storage system that ensures file assured deletion and works seamlessly atop today's cloud storage services. FADE decouples the management of encrypted data and encryption keys, such that encrypted data remains on third-party (untrusted) cloud storage providers, while encryption keys are independently maintained by a key manager service, whose trustworthiness can be enforced using a quorum scheme [18]. FADE generalizes time-based file assured deletion [5, 14] (i.e., files are assuredly deleted upon time expiration) into a more fine-grained approach called policybased file assured deletion, in which files are associated with more flexible file access policies (e.g., time expiration, read/write permissions of authorized users) and are assuredly deleted when the associated file access policies are revoked and become obsolete.

A motivating application of FADE is cloud-based backup systems (e.g., JungleDisk [7], Cumulus [21]), which use the cloud as the backup storage for files. FADE can be viewed as a value-added security service that further enhances the security properties of the existing cloud-based backup systems.

In summary, our paper makes the following contributions:

- We propose a new policy-based file assured deletion scheme that reliably deletes files with regard to revoked file access policies. In this context, we design the key management schemes for various file manipulation operations.

- We implement a working prototype of FADE atop Amazon S3 [2]. Our implementation aims to illustrate that various applications can benefit from FADE, such as cloud-based backup systems. FADE consists of a set of API interfaces that we can export, so that we can adapt FADE into different cloud storage implementations. 
- We empirically evaluate the performance overhead of FADE atop Amazon S3, and using realistic experiments, we show the feasibility of FADE in improving the security protection of data storage on the cloud.

The remainder of the paper proceeds as follows. In Section 2, we present the design of policy-base file assured deletion, a major building block of FADE. In Section 3, we explain the implementation details of FADE. In Section 4, we evaluate FADE atop Amazon S3. Section 5 discusses the limitations of FADE and possible enhancements. In Section 6, we review related work on protecting outsourced data storage. Finally, Section 7 concludes.

\section{Policy-based File Assured Deletion}

We present policy-based file assured deletion, the major design building block of our FADE architecture. Our main focus is to deal with the cryptographic key operations that enable file assured deletion. We first review time-based file assured deletion. We then explain how it can be extended to policy-based file assured deletion.

\subsection{Background}

Time-based file assured deletion, which is first introduced in [14], means that files can be securely deleted and remain permanently inaccessible after a predefined duration. The main idea is that a file is encrypted with a data key, and this data key is further encrypted with a control key that is maintained by a separate key manager service (known as Ephemerizer [14]). In [14], the control key is time-based, meaning that it will be completely removed by the key manager when an expiration time is reached, where the expiration time is specified when the file is first declared. Without the control key, the data key and hence the data file remain encrypted and are deemed to be inaccessible. Thus, the main security property of file assured deletion is that even if a cloud provider does not remove expired file copies from its storage, those files remain encrypted and unrecoverable.

Time-based file assured deletion is later prototyped in Vanish [5]. Vanish divides a data key into multiple key shares, which are then stored in different nodes of a peer-to-peer network. Nodes remove the key shares that reside in their caches for 8 hours. If a file needs to remain accessible after 8 hours, then the file owner needs to update the key shares in node caches.

However, both [14] and [5] target only the assured deletion upon time expiration, and do not consider a more fine-grained control of assured deletion with respect to different file access policies. We elaborate this issue in Section 2.2.

\subsection{Policy-based Deletion}

We associate each file with a single atomic file access policy (or policy for short), or more generally, a Boolean combination of atomic policies. Each (atomic) policy 
is associated with a control key, and all the control keys are maintained by the key manager. Similar to time-based deletion, the file content is encrypted with a data key, and the data key is further encrypted with the control keys corresponding to the policy combination. When a policy is revoked, the corresponding control key will be removed from the key manager. Thus, when the policy combination associated with a file is revoked and no longer holds, the data key and hence the encrypted content of the file cannot be recovered with the control keys of the policies. In this case, we say the file is deleted. The main idea of policy-based deletion is to delete files that are associated with revoked policies.

The definitions of policies vary depending on applications. Time-based deletion is a special case under our framework, and policies with other access rights can be defined. To motivate the use of policy-based deletion, let us consider a scenario where a company outsources its data to the cloud. We consider four practical cases where policy-based deletion will be useful:

- Storing files for tenured employees. For each employee (e.g., Alice), we can define a user-based policy "P: Alice is an employee", and associate this policy with all files of Alice. If Alice quits her job, then the key manager will expunge the control key of policy $P$. Thus, nobody including Alice can access the files associated with $P$ on the cloud, and those files are said to be deleted.

- Storing files for contract-based employees. An employee may be affiliated with the company for only a fixed length of time. Then we can form a combination of the user-based and time-based policies for employees' files. For example, for a contract-based employee Bob whose contract expires on 201001-01, we have two policies " $P_{1}: B o b$ is an employee" and " $P_{2}$ : valid before 2010-01-01". Then all files of Bob are associated with the policy combination $P_{1} \wedge P_{2}$. If either $P_{1}$ or $P_{2}$ is revoked, then Bob's files are deleted.

- Storing files for a team of employees. The company may have different teams, each of which has more than one employee. As in above, we can assign each employee $i$ a policy combination $P_{i 1} \wedge P_{i 2}$, where $P_{i 1}$ and $P_{i 2}$ denote the user-based and time-based policies, respectively. We then associate the team's files with the disjunctive combination $\left(P_{11} \wedge P_{12}\right) \vee\left(P_{21} \wedge P_{22}\right) \vee \cdots \vee\left(P_{N 1} \wedge P_{N 2}\right)$ for employees $1,2, \ldots, N$. Thus, the team's files can be accessed by any one of the employees, and will be deleted when the policies of all employees of the team are revoked.

- Switching a cloud provider. The company can define a customer-based policy " $P$ : a customer of cloud provider $X$ ", and all files that are stored on cloud $X$ are tied with policy $P$. If the company switches to a new cloud provider, then it can revoke policy $P$. Thus, all files on cloud $X$ will be deleted.

Policy-based deletion follows the similar notion of attribute-based encryption $(A B E)[6,16,17]$, in which data can be accessed only if a subset of attributes (policies) are satisfied. However, our work is different from ABE in two aspects. First, we focus on how to delete data, while ABE focuses on how to access data based on attributes. Second, because of the different design objectives, ABE gives users the decryption keys of the associated attributes, so that they can access files that satisfy the attributes. On the other hand, in policy-based deletion, we 
do not share with users any decryption keys of policies, which instead are all maintained in the key manager. Our focus is to appropriately remove keys in the key manager so as to guarantee file assured deletion, which is an important security property when we outsource data storage to the cloud. This guides us into a different design space in contrast with existing $\mathrm{ABE}$ approaches.

\subsection{Participants in the System}

Our system is composed of three participants: the data owner, the key manager, and the storage cloud. They are described as follows.

Data owner. The data owner is the entity that originates file data to be stored on the cloud. It may be a file system of a PC, a user-level program, a mobile device, or even in the form of a plug-in of a client application.

Key manager. The key manager maintains the policy-based control keys that are used to encrypt data keys. It responds to the data owner's requests by performing encryption, decryption, renewal, and revocation to the control keys.

Storage cloud. The storage cloud is maintained by a third-party cloud provider (e.g., Amazon S3) and keeps the data on behalf of the data owner. We emphasize that we do not require any protocol and implementation changes on the storage cloud to support our system. Even a naive storage service that merely provides file upload/download operations will be suitable.

\subsection{Threat Models and Assumptions}

Our main design goal is to provide assured deletion of files produced by the data owner. A file is deleted (or permanently inaccessible) if its policy is revoked and becomes obsolete. Here, we assume that the control key associated with a revoked policy is reliably removed by the key manager. Thus, by assured deletion of files, we mean that any existing file copy that are associated with revoked policies will remain permanently encrypted and unrecoverable.

The key manager can be deployed as a minimally trusted third-party service. By minimally trusted, we mean that the key manager reliably removes the control keys of revoked policies. However, it is possible that the key manager can be compromised. In this case, an attacker can recover the files that are associated with existing active policies. On the other hand, files that are associated with revoked policies still remain inaccessible, as the control keys are removed. Hence, file assured deletion is achieved.

It is still important to improve the robustness of the key manager service to minimize its chance of being compromised. To achieve this, we can use a quorum of key managers [18], in which we create $n$ key shares for a key, such that any $k<n$ of the key shares can be used to recover the key. While the quorum scheme increases the storage overhead of keys, this is justified as keys are of much smaller size than data files.

Before accessing the active keys in the key manager, the data owner needs to present authentication credentials (e.g., based on public key infrastructure 
certificates) to the key manager to show that it satisfies the proper policies associated with the files. We assume that the data owner does not disclose any successfully decrypted file to unauthorized parties.

\subsection{The Basics - File Upload/Download}

We now introduce the basics of uploading/downloading files to/from the cloud storage. We first assume that each file is associated with a single policy, and then explain how a file is associated with multiple policies in Section 2.7.

Our design is based on blinded $R S A[14,20]$, in which the data owner requests the key manager to decrypt a blinded version of the encrypted data key. If the associated policy is satisfied, then the key manager will decrypt and return the blinded version of the original data key. The data owner can then recover the data key. In this way, the actual content of the data key remains confidential to the key manager as well as to any attacker that sniffs the communication between the data owner and the key manager.

We first summarize the major notation used throughout the paper. For each policy $i$, the key manager generates two secret large RSA prime numbers $p_{i}$ and $q_{i}$ and computes the product $n_{i}=p_{i} q_{i}{ }^{1}$. The key manager then randomly chooses the RSA public-private control key pair $\left(e_{i}, d_{i}\right)$. The parameters $\left(n_{i}, e_{i}\right)$ will be publicized, while $d_{i}$ is securely stored in the key manager. On the other hand, when the data owner encrypts a file $F$, it randomly generates a data key $K$, and a secret key $S_{i}$ that corresponds to policy $P_{i}$. We let $\{m\}_{k}$ denote a message $m$ encrypted with key $k$ using symmetric-key encryption (e.g., AES). We let $R$ be the blinded component when we use blinded RSA for the exchanges of cryptographic keys.

Suppose that $F$ is associated with policy $P_{i}$. Our goal here is to ensure that $K$, and hence $F$, are accessible only when policy $P_{i}$ is satisfied. Note that we only present the operations on cryptographic keys, while the implementation subtleties, such as metadata, will be discussed in Section 3. Also, when we raise some number to exponents $e_{i}$ or $d_{i}$, it must be done over modulo $n_{i}$. For brevity, we drop "mod $n_{i}$ " in our discussion.

File upload. Figure 1 shows the file upload operation. The data owner first requests the public key $\left(n_{i}, e_{i}\right)$ of policy $P_{i}$ from the key manager, and caches $\left(n_{i}, e_{i}\right)$ for subsequent uses if the same policy $P_{i}$ is associated with other files. Then the data owner generates two random keys $K$ and $S_{i}$, and sends $\{K\}_{S_{i}}$, $S_{i}^{e_{i}}$, and $\{F\}_{K}$ to the cloud ${ }^{2}$. Then the data owner can discard $K$ and $S_{i}$.

File download. Figure 2 shows the file download operation. The data owner fetches $\{K\}_{S_{i}}, S_{i}^{e_{i}}$, and $\{F\}_{K}$ from the storage cloud. Then the data owner generates a secret random number $R$, computes $R^{e_{i}}$, and sends $S_{i}^{e_{i}} \cdot R^{e_{i}}=\left(S_{i} R\right)^{e_{i}}$ to the key manager to request for decryption. The key manager then computes

\footnotetext{
${ }^{1}$ We require that each policy $i$ uses a distinct $n_{i}$ to avoid the common modulus attack on RSA [10].

${ }^{2}$ We point out that the encrypted keys (i.e., $\{K\}_{S_{i}}, S_{i}^{e_{i}}$ ) can be stored in the cloud without creating risks of leaking confidential information.
} 
FADE: Secure Overlay Cloud Storage with File Assured Deletion

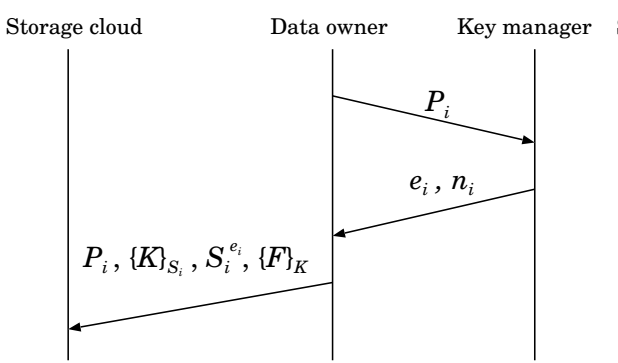

Fig. 1: File upload.

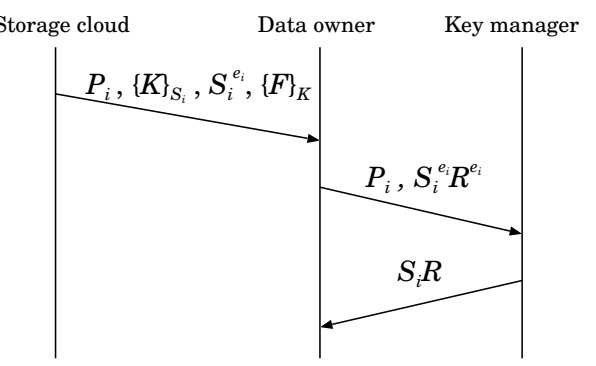

Fig. 2: File download.

and returns $\left(\left(S_{i} R\right)^{e_{i}}\right)^{d_{i}}=S_{i} R$ to the data owner. The data owner can now remove $R$ and obtain $S_{i}$, and decrypt $\{K\}_{S_{i}}$ and hence $\{F\}_{K}$.

Integrity. To protect the integrity of a file, the data owner needs to compute an HMAC on every encrypted file and stores the HMAC, together with the encrypted file, in the cloud storage. When a file is downloaded, the data owner will check whether the HMAC is valid before decrypting the file. We assume that the data owner has a long-term private secret for the HMAC computation.

\subsection{Policy Revocation for File Assured Deletion}

If a policy $P_{i}$ is revoked, then the key manager completely removes the private key $d_{i}$ and the secret prime numbers $p_{i}$ and $q_{i}$. Thus, we cannot recover $S_{i}$ from $S_{i}^{e_{i}}$, and hence cannot recover $K$ and the file $F$. We say that the file $F$, which is tied to policy $P_{i}$, is assuredly deleted. Note that the policy revocation operations do not involve interactions with the storage cloud.

\subsection{Multiple Policies}

In addition to one policy per file, FADE supports a Boolean combination of multiple policies. We mainly focus on two kinds of logical connectives: (i) the conjunction (AND), which means the data is accessible only when every policy is satisfied; and (ii) the disjunction (OR), which means if any policy is satisfied, then the data is accessible.

- Conjunctive Policies. Suppose that $F$ is associated with conjunctive policies $P_{1} \wedge P_{2} \wedge \cdots \wedge P_{m}$. To upload $F$ to the storage cloud, the data owner first randomly generates a data key $K$, and secret keys $S_{1}, S_{2}, \ldots, S_{m}$. It then sends the following to the storage cloud: $\left\{\{K\}_{S_{1}}\right\}_{S_{2}} \cdots_{S_{m}}, S_{1}^{e_{1}}, S_{2}^{e_{2}}, \ldots$, $S_{m}^{e_{m}}$, and $\{F\}_{K}$. On the other hand, to recover $F$, the data owner generates a random number $R$ and sends $\left(S_{1} R\right)^{e_{1}},\left(S_{2} R\right)^{e_{2}}, \ldots,\left(S_{m} R\right)^{e_{m}}$ to the key manager, which then returns $S_{1} R, S_{2} R, \ldots, S_{m} R$. The data owner can then recover $S_{1}, S_{2}, \ldots, S_{m}$, and hence $K$ and $F$.

- Disjunctive Policies. Suppose that $F$ is associated with disjunctive policies $P_{i_{1}} \vee P_{i_{2}} \vee \cdots \vee P_{i_{m}}$. To upload $F$ to the cloud, the data owner will send the 
following: $\{K\}_{S_{1}},\{K\}_{S_{2}}, \ldots,\{K\}_{S_{m}}, S_{1}^{e_{1}}, S_{2}^{e_{2}}, \ldots, S_{m}^{e_{m}}$, and $\{F\}_{K}$. Therefore, the data owner needs to compute $m$ different encrypted copies of $K$. On the other hand, to recover $F$, we can use any one of the policies to decrypt the file, as in the above operations.

To delete a file associated with conjunctive policies, we simply revoke any of the policies (say, $P_{j}$ ). Thus, we cannot recover $S_{j}$ and hence the data key $K$ and file $F$. On the other hand, to delete a file associated with disjunctive policies, we need to revoke all policies, so that $S_{j}^{e_{j}}$ cannot be recovered for all $j$. Note that for any Boolean combination of policies, we can express it in canonical form, e.g., in the disjunction (OR) of conjunctive (AND) policies.

\subsection{Policy Renewal}

We conclude this section with the discussion of policy renewal. Policy renewal means to associate a file with a new policy (or combination of policies). For example, if a user wants to extend the expiration time of a file, then the user can update the old policy that specifies an earlier expiration time to the new policy that specifies a later expiration time. However, to guarantee file assured deletion, policy renewal can be performed only when the following condition holds: the old policy will always be revoked first before the new policy is revoked. The reason is that after policy renewal, there will be two versions of a file: one is protected with the old policy, and one is protected with the new policy. If the new policy is revoked first, then the file version that is protected with the old policy may still be accessible when the control keys of the old policy are compromised, meaning that the file is not assuredly deleted.

It is important to note that it is a non-trivial task to enforce the condition of policy renewal, as the old policy may be associated with other existing files. In this paper, we do not consider this issue and we pose it as future work.

Suppose that we have enforced the condition of policy renewal. A straightforward approach of implementing policy renewal is to combine the file upload and download operations, but without retrieving the encrypted file from the cloud. The procedures can be summarized as follows: (i) download all encrypted keys from the storage cloud, (ii) send them to the key manager for decryption, (iii) recover the data key, (iv) re-encrypt the data key with the control keys of the new policies, and finally (v) send the newly encrypted keys back to the cloud.

In some special cases, optimization can be made so as to save the operations of decrypting and re-encrypting the data key. Suppose that the Boolean combination structure of policies remain unchanged, but one of the atomic policies $P_{i}$ is changed $P_{i^{\prime}}$. For example, when we extend the contract date of Bob (see Section 2.2), we may need to update the particular time-based policy of Bob without changing other policies. In this case, the data owner simply sends the blinded version $S_{i}{ }^{e_{i}} R^{e_{i}}$ to the key manager, which then returns $S_{i} R$. The data owner then recovers $S_{i}$. Now, the data owner re-encrypts $S_{i}$ into $S_{i}^{e_{i^{\prime}}}\left(\bmod n_{i^{\prime}}\right)$, where $\left(n_{i^{\prime}}, e_{i^{\prime}}\right)$ is the public key of policy $P_{i^{\prime}}$, and sends it to the cloud. Note 


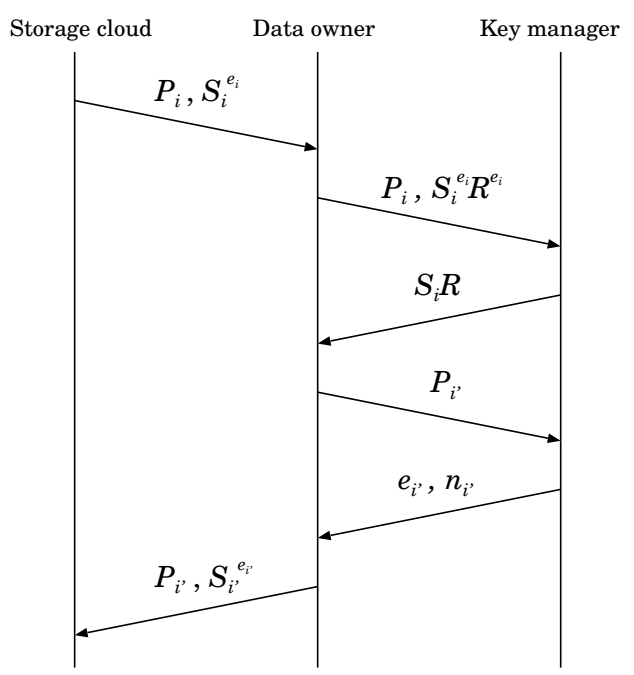

Fig. 3: Policy renewal.

that the encrypted data key $K$ remains intact. Figure 3 illustrates this special case of policy renewal.

\section{The FADE Architecture}

We implement a working prototype of FADE using $\mathrm{C}++$ on Linux, and we use the OpenSSL library [13] for the cryptographic operations. In addition, we use Amazon S3 [2] as our storage cloud. This section is to address the implementation issues of our FADE architecture, based on our experience in prototyping FADE. Our goal is to show the practicality of FADE when it is deployed with today's cloud storage services.

Figure 4 shows the FADE architecture. In the following, we define the metadata of FADE attached to individual files. We then describe how we implement the data owner and the key manager, and how the data owner interacts with the storage cloud.

\subsection{Representation of Metadata}

For each file protected by FADE, we include the metadata that describes the policies associated with the file as well as a set of encrypted keys. In FADE, there are two types of metadata: file metadata and policy metadata.

File metadata. The file metadata mainly contains two pieces of information: file size and HMAC. We hash the encrypted file with HMAC-SHA1 for integrity checking. The file metadata is of fixed size (with 8 bytes of file size and 20 bytes of HMAC) and attached at the beginning of the encrypted file. Both the file 


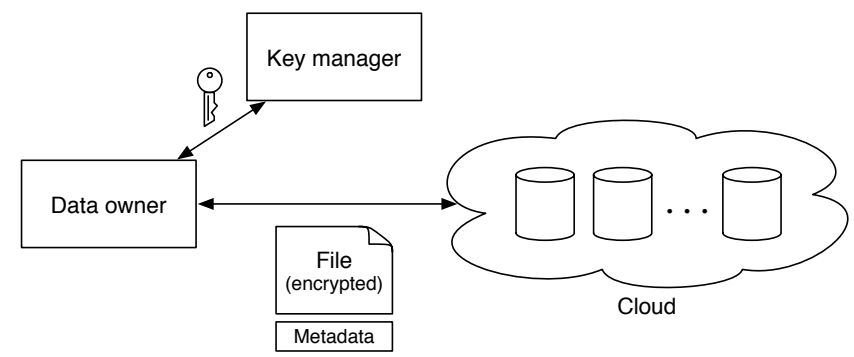

Fig. 4: The FADE architecture.

metadata and the encrypted data file will then be treated as a single file to be uploaded to the storage cloud.

Policy metadata. The policy metadata includes the specification of the Boolean combination of policies and the corresponding encrypted cryptographic keys. Here, we assume that each single policy is specified by a unique 4-byte integer identifier. To represent a Boolean combination of policies, we express it in disjunctive canonical form, i.e., the disjunction (OR) of conjunctive policies, and use the characters ' $*$ ' and ' + ' to denote the AND and OR operators. Then we upload the policy metadata as a separate file to the storage cloud. This enables us to renew policies directly on the policy metadata without retrieving the entire file from the storage cloud.

In our implementation, individual files have their own policy metadata, although we allow multiple files to be associated with the same policy (which is the expected behavior of FADE). In other words, for two data files that are under the same policy, they will have different policy metadata files that specify different data keys, and the data keys are protected by the control key of the same policy. In Section 5, we discuss how we may associate the same policy metadata file with multiple data files so as to reduce the metadata overhead.

\subsection{Data Owner and Storage Cloud}

Our implementation of the data owner uses the following four function calls to enable end users to interact with the storage cloud:

- Upload(file, policy). The data owner encrypts the input file using the specified policy (or a Boolean combination of policies). It then sends the encrypted file and the metadata onto the cloud. In our implementation, the file is encrypted using the 128-bit AES algorithm with the cipher block chaining (CBC) mode, yet we can adopt a different symmetric-key encryption algorithm depending on applications.

- Download(file). The data owner retrieves the file and the policy metadata from the cloud, checks the integrity of the file, and decrypts the file.

- Delete(policy). The data owner tells the key manager to permanently revoke the specified policy. All files associated with the policy will be assuredly deleted. 
- Renew(file, new_policy). The data owner first fetches the policy metadata for the given file from the cloud. It then updates the policy metadata with the new policy. Finally, it sends the policy metadata back to the cloud.

The above function calls can be exported as library APIs that can be embedded into different implementations of the data owner. In our current prototype, we implement the data owner as a user-level program that can access files under a working directory of a desktop PC.

The above exported interfaces wrap the third-party APIs for interacting with the storage cloud. As an example, we use LibAWS++ [9], a C++ library for interfacing with Amazon S3. We note that the LibAWS++ library uses HTTP to communicate with the cloud, and it does not provide any security protection on the data being transferred. To interact with different cloud storage services, we can use different third-party APIs, provided that the APIs support the basic file upload/download operations.

\subsection{Key Manager}

We implement the key manager that supports the following four basic functions.

- Creating a policy. The key manager creates a new policy and returns the corresponding public control key.

- Retrieving the public control key of a policy. If the policy is accessible, then the key manager returns the public control key. Otherwise, it returns an error.

- Decrypting a key with respect to a policy. If the policy is accessible, then the key manager decrypts the (blinded) key. Otherwise, it returns an error.

- Revoking a policy. The key manager revokes the policy and removes the corresponding keys.

We implement the basic functionalities of the key manager so that it can perform the required operations on the cryptographic keys. In particular, all the policy control keys are built upon 1024-bit blinded RSA (see Section 2.5). To make the key manager more robust, we can extend the key manager to a quorum of key managers as stated in [18], and implement a PKI-based certification system for policy checking (see Section 2.4).

\section{Evaluation}

We implement a prototype of FADE atop Amazon S3 [2], and we now evaluate the empirical performance of FADE. It is crucial that FADE does not introduce substantial performance overhead that will lead to a big increase in data management costs. In addition, the cryptographic operations of FADE should only bring insignificant computational overhead. Therefore, our experiments aim to answer the following issue: What is the performance overhead of FADE, and is it feasible to use FADE to provide file assured deletion for cloud storage? 
Our experiments use Amazon S3, residing in the United States, as the storage cloud. Also, we deploy the data owner and the key manager within an organization's network that resides in an Asian country. In the experiments, we evaluate FADE when it operates on an individual file of different sizes: 1KB, $10 \mathrm{~KB}, 100 \mathrm{~KB}, 1 \mathrm{MB}$, and $10 \mathrm{MB}$.

\subsection{Experimental Results on Time Performance of FADE}

We now measure the time performance of FADE using our prototype. In order to identify the time overhead of FADE, we divide the running time of each measurement into three components:

- data transmission time, the data uploading/downloading time between the data owner and the storage cloud. We further divide it into two components: the file component, which measures the transmission time for the file body and the file metadata, and the policy component, which measures the transmission time for the policy metadata (see Section 3.1). We upload/download these two components as two separate copies to/from the storage cloud.

- AES and HMAC time, the total computational time used for performing AES and HMAC on the file.

- key management time, the time for the data owner to coordinate with the key manager on operating the cryptographic keys. For the file upload operation (see Figure 1 in Section 2.5), we require the data owner to obtain the public control key for the corresponding policy; for the download operation (see Figure 2 in Section 2.5), the data owner works with the key manager to obtain the data key.

We average each of our measurement results over 10 different trials.

Experiment 1 (Performance of file upload/download operations).

First, we measure the running time of the file upload and download operations for different file sizes. Table 1 shows the results. We find that the transmission time is the dominant factor (over 99\%). The AES and HMAC time increases linearly with the file size. However, the key management time stays constant on the order of milliseconds, regardless of the file size. In other words, compared with the basic encryption and integrity check provided by AES and HMAC, FADE only involves a small time overhead in key management.

We note that when the file size is small, the transmission time for the policy metadata is comparable with the transmission time for the file. To understand this, we capture and analyze the data traffic, and find that the round-trip time between our network (in Asia) and Amazon S3 (in the United States) is 200300 milliseconds. Because the file and the policy metadata are stored on the cloud as two separate copies, they are transferred through two different TCP connections, and a significant portion of data transmission time is actually due to the TCP connection setup. In Section 4.2, we will show that the actual number of bytes stored for the policy metadata is in fact much less than that for the file.

Experiment 2 (Performance of policy updates). Table 2 shows the time used for renewing a single policy of a file (see Figure 3 in Section 2.8), in 


\begin{tabular}{|c|c|cc|cc|cc|cc|}
\hline \multirow{2}{*}{ File size } & \multirow{2}{*}{ Total time } & \multicolumn{3}{|c|}{ Data transmission } & \multicolumn{3}{|c|}{ AES+HMAC } & \multicolumn{2}{|c|}{ Key management } \\
\cline { 3 - 9 } & & File & $(\%)$ & Policy & $(\%)$ & Time & $(\%)$ & Time & $(\%)$ \\
\hline $1 \mathrm{~KB}$ & $1.260 \mathrm{~s}$ & $0.724 \mathrm{~s}$ & $57.4 \%$ & $0.537 \mathrm{~s}$ & $42.6 \%$ & $0.000 \mathrm{~s}$ & $0.0 \%$ & $0.000 \mathrm{~s}$ & $0.0 \%$ \\
$10 \mathrm{~KB}$ & $1.552 \mathrm{~s}$ & $1.020 \mathrm{~s}$ & $65.7 \%$ & $0.532 \mathrm{~s}$ & $34.3 \%$ & $0.001 \mathrm{~s}$ & $0.0 \%$ & $0.000 \mathrm{~s}$ & $0.0 \%$ \\
$100 \mathrm{~KB}$ & $2.452 \mathrm{~s}$ & $1.903 \mathrm{~s}$ & $77.6 \%$ & $0.546 \mathrm{~s}$ & $22.3 \%$ & $0.002 \mathrm{~s}$ & $0.1 \%$ & $0.001 \mathrm{~s}$ & $0.0 \%$ \\
$1 \mathrm{MB}$ & $4.194 \mathrm{~s}$ & $3.646 \mathrm{~s}$ & $86.9 \%$ & $0.527 \mathrm{~s}$ & $12.6 \%$ & $0.022 \mathrm{~s}$ & $0.5 \%$ & $0.000 \mathrm{~s}$ & $0.0 \%$ \\
$10 \mathrm{MB}$ & $16.275 \mathrm{~s}$ & $15.463 \mathrm{~s}$ & $95.0 \%$ & $0.595 \mathrm{~s}$ & $3.7 \%$ & $0.218 \mathrm{~s}$ & $1.3 \%$ & $0.000 \mathrm{~s}$ & $0.0 \%$ \\
\hline
\end{tabular}

(a) Upload

\begin{tabular}{|c|c|cc|cc|cc|cc|}
\hline \multirow{2}{*}{ File size } & \multirow{2}{*}{ Total time } & \multicolumn{3}{|c|}{ Data transmission } & \multicolumn{3}{|c|}{ AES+HMAC } & \multicolumn{2}{|c|}{ Key management } \\
\cline { 3 - 8 } & & File & $(\%)$ & Policy & $(\%)$ & Time & $(\%)$ & Time & $(\%)$ \\
\hline $1 \mathrm{~KB}$ & $0.843 \mathrm{~s}$ & $0.485 \mathrm{~s}$ & $57.5 \%$ & $0.355 \mathrm{~s}$ & $42.1 \%$ & $0.000 \mathrm{~s}$ & $0.0 \%$ & $0.003 \mathrm{~s}$ & $0.4 \%$ \\
$10 \mathrm{~KB}$ & $0.912 \mathrm{~s}$ & $0.615 \mathrm{~s}$ & $67.4 \%$ & $0.294 \mathrm{~s}$ & $32.2 \%$ & $0.000 \mathrm{~s}$ & $0.0 \%$ & $0.003 \mathrm{~s}$ & $0.3 \%$ \\
$100 \mathrm{~KB}$ & $1.968 \mathrm{~s}$ & $1.682 \mathrm{~s}$ & $85.5 \%$ & $0.282 \mathrm{~s}$ & $14.3 \%$ & $0.002 \mathrm{~s}$ & $0.1 \%$ & $0.002 \mathrm{~s}$ & $0.1 \%$ \\
$1 \mathrm{MB}$ & $4.696 \mathrm{~s}$ & $4.360 \mathrm{~s}$ & $92.8 \%$ & $0.317 \mathrm{~s}$ & $6.7 \%$ & $0.017 \mathrm{~s}$ & $0.4 \%$ & $0.002 \mathrm{~s}$ & $0.1 \%$ \\
$10 \mathrm{MB}$ & $33.746 \mathrm{~s}$ & $33.182 \mathrm{~s}$ & $98.3 \%$ & $0.395 \mathrm{~s}$ & $1.2 \%$ & $0.166 \mathrm{~s}$ & $0.5 \%$ & $0.002 \mathrm{~s}$ & $0.0 \%$ \\
\hline
\end{tabular}

(b) Download

Table 1: Experiment 1 (Performance of upload/download operations).

\begin{tabular}{|c|c|cc|cc|cc|}
\hline \multirow{2}{*}{ File size } & \multirow{2}{*}{ Total time } & \multicolumn{4}{|c|}{ Data transmission } & \multicolumn{2}{|c|}{ Key management } \\
\cline { 3 - 7 } & & Download & $(\%)$ & Upload & $(\%)$ & Time & $(\%)$ \\
\hline $1 \mathrm{~KB}$ & $0.923 \mathrm{~s}$ & $0.315 \mathrm{~s}$ & $34.1 \%$ & $0.605 \mathrm{~s}$ & $65.5 \%$ & $0.004 \mathrm{~s}$ & $0.4 \%$ \\
$10 \mathrm{~KB}$ & $0.805 \mathrm{~s}$ & $0.266 \mathrm{~s}$ & $33.0 \%$ & $0.536 \mathrm{~s}$ & $66.6 \%$ & $0.004 \mathrm{~s}$ & $0.4 \%$ \\
$100 \mathrm{~KB}$ & $0.821 \mathrm{~s}$ & $0.271 \mathrm{~s}$ & $33.0 \%$ & $0.546 \mathrm{~s}$ & $66.5 \%$ & $0.004 \mathrm{~s}$ & $0.5 \%$ \\
$1 \mathrm{MB}$ & $0.813 \mathrm{~s}$ & $0.273 \mathrm{~s}$ & $33.5 \%$ & $0.537 \mathrm{~s}$ & $66.0 \%$ & $0.003 \mathrm{~s}$ & $0.4 \%$ \\
$10 \mathrm{MB}$ & $0.832 \mathrm{~s}$ & $0.266 \mathrm{~s}$ & $32.0 \%$ & $0.562 \mathrm{~s}$ & $67.6 \%$ & $0.004 \mathrm{~s}$ & $0.5 \%$ \\
\hline
\end{tabular}

Table 2: Experiment 2 (Performance of policy updates). We do not show the $\mathrm{AES}+\mathrm{HMAC}$ time as it is not involved in policy renewal.

which we update the policy metadata on the storage cloud with the new set of cryptographic keys. Our experiments show that the total time is generally small (less than a second) regardless of the file size, as we operate on the policy metadata only. Also, the key management time only takes about $0.004 \mathrm{~s}$ in renewing a policy, and this value is again independent of the file size.

Experiment 3 (Performance of multiple policies). We now evaluate the performance of FADE when multiple policies are associated with a file (see Section 2.7). Here, we focus on the file upload operation, and fix the file size at $1 \mathrm{MB}$. We look at two specific combinations of policies, one on the conjunctive case and one on the disjunctive case.

Table 3a shows different components of time for different numbers of conjunctive policies, and Table $3 \mathrm{~b}$ shows the case for disjunctive policies. A key observation is that the AES and HMAC and the key management time remain very low (on the order of milliseconds) when the number of policies increases. 


\begin{tabular}{|c|c|c|c|c|c|c|c|}
\hline \multirow{2}{*}{$\begin{array}{c}\text { Number of } \\
\text { policies }\end{array}$} & \multirow{2}{*}{ Total time } & \multicolumn{2}{|c|}{ Data transmission } & \multicolumn{4}{|c|}{\begin{tabular}{|l|l|} 
AES+HMAC & Key management
\end{tabular}} \\
\hline & & File $\quad(\%)$ & Policy (\%) & Time & $(\%)$ & Time & $(\%)$ \\
\hline 1 & $5.141 \mathrm{~s}$ & $4.562 \mathrm{~s} 88.7 \%$ & $0.557 \mathrm{~s} 10.8 \%$ & $0.022 \mathrm{~s}$ & $0.4 \%$ & $0.000 \mathrm{~s}$ & $0.0 \%$ \\
\hline 2 & $4.970 \mathrm{~s}$ & $4.352 \mathrm{~s} 87.6 \%$ & $0.595 \mathrm{~s} 12.0 \%$ & $0.022 \mathrm{~s}$ & $0.4 \%$ & $0.000 \mathrm{~s}$ & $0.0 \%$ \\
\hline 3 & $4.667 \mathrm{~s}$ & $3.983 \mathrm{~s} 85.3 \%$ & $0.662 \mathrm{~s} 14.2 \%$ & $0.022 \mathrm{~s}$ & $0.5 \%$ & $0.001 \mathrm{~s}$ & $0.0 \%$ \\
\hline 4 & $4.976 \mathrm{~s}$ & $4.397 \mathrm{~s} 88.4 \%$ & $0.557 \mathrm{~s} 11.2 \%$ & $0.022 \mathrm{~s}$ & $0.4 \%$ & $0.001 \mathrm{~s}$ & $0.0 \%$ \\
\hline 5 & $4.962 \mathrm{~s}$ & $4.406 \mathrm{~s} 88.8 \%$ & $0.533 \mathrm{~s} 10.7 \%$ & $0.021 \mathrm{~s}$ & $0.4 \%$ & $0.001 \mathrm{~s}$ & $0.0 \%$ \\
\hline
\end{tabular}

(a) Conjunctive policies

\begin{tabular}{|c|c|cc|cc|cc|cc|}
\hline \multirow{2}{*}{$\begin{array}{c}\text { Number of } \\
\text { policies }\end{array}$} & \multirow{2}{*}{ Total time } & \multicolumn{3}{|c|}{ Data transmission } & \multicolumn{2}{|c|}{ AES+HMAC } & \multicolumn{2}{|c|}{ Key management } \\
\cline { 3 - 8 } & & File (\%) & Policy & $(\%)$ & Time & $(\%)$ & Time & $(\%)$ \\
\hline 1 & $3.927 \mathrm{~s}$ & $3.364 \mathrm{~s}$ & $85.7 \%$ & $0.541 \mathrm{~s}$ & $13.8 \%$ & $0.022 \mathrm{~s}$ & $0.6 \%$ & $0.000 \mathrm{~s}$ & $0.0 \%$ \\
2 & $4.015 \mathrm{~s}$ & $3.460 \mathrm{~s}$ & $86.2 \%$ & $0.534 \mathrm{~s}$ & $13.3 \%$ & $0.021 \mathrm{~s}$ & $0.5 \%$ & $0.000 \mathrm{~s}$ & $0.0 \%$ \\
3 & $3.923 \mathrm{~s}$ & $3.390 \mathrm{~s}$ & $86.4 \%$ & $0.511 \mathrm{~s}$ & $13.0 \%$ & $0.022 \mathrm{~s}$ & $0.6 \%$ & $0.001 \mathrm{~s}$ & $0.0 \%$ \\
4 & $3.859 \mathrm{~s}$ & $3.322 \mathrm{~s}$ & $86.1 \%$ & $0.515 \mathrm{~s}$ & $13.3 \%$ & $0.022 \mathrm{~s}$ & $0.6 \%$ & $0.000 \mathrm{~s}$ & $0.0 \%$ \\
5 & $4.118 \mathrm{~s}$ & $3.559 \mathrm{~s}$ & $86.4 \%$ & $0.536 \mathrm{~s}$ & $13.0 \%$ & $0.022 \mathrm{~s}$ & $0.5 \%$ & $0.001 \mathrm{~s}$ & $0.0 \%$ \\
\hline
\end{tabular}

(b) Disjunctive policies

Table 3: Experiment 3 (Performance of multiple policies).

\begin{tabular}{|c|c|}
\hline $\begin{array}{c}\text { Number of } \\
\text { policies }\end{array}$ & $\begin{array}{c}\text { Policy metadata } \\
\text { size (bytes) }\end{array}$ \\
\hline 1 & 149 \\
2 & 282 \\
3 & 415 \\
4 & 548 \\
5 & 681 \\
\hline
\end{tabular}

(a) Conjunctive policies

\begin{tabular}{|c|c|}
\hline $\begin{array}{c}\text { Number of } \\
\text { policies }\end{array}$ & $\begin{array}{c}\text { Policy metadata } \\
\text { size (bytes) }\end{array}$ \\
\hline 1 & 149 \\
2 & 298 \\
3 & 447 \\
4 & 596 \\
5 & 745 \\
\hline
\end{tabular}

(b) Disjunctive policies

Table 4: Size of the policy metadata.

\subsection{Space Utilization of FADE}

We now assess the space utilization. As stated in Section 3.1, there are file metadata and policy metadata for each file, and this metadata information is the space overhead introduced by FADE. For the file metadata, it is always fixed at 28 bytes. On the other hand, for the policy metadata, its size differs with the number of policies. For instance, we need 128 bytes for the policy-based secret key $S_{i}^{e_{i}}$ for some policy $i$. The size of an encrypted copy of $K$ is 16 bytes, and this size increases with the number of terms in the case of disjunctive (OR) policies (see Section 2.7). Table 4 shows the different sizes of the policy metadata based on our implementation prototype for a variable number of (a) conjunctive policies $\left(P_{1} \wedge P_{2} \wedge \cdots \wedge P_{m}\right)$, and (b) disjunctive policies $\left(P_{1} \vee P_{2} \vee \cdots \vee P_{m}\right)$. For instance, if the file size is $1 \mathrm{MB}$ and there is only one policy, then the size of the file metadata is 28 bytes and the policy metadata is 149 bytes, and hence the space overhead is $0.017 \%$. 


\subsection{Lessons Learned}

In this section, we evaluate the performance of FADE in terms of the overheads of time, space utilization, and data transfer. It is important to note that the performance results depend on the deployment environment. For instance, if the data owner and the key manager all reside in the United States as Amazon S3, then the transmission times for files and metadata will significantly reduce; or if the policy metadata contains more descriptive information, the overhead will increase. Nevertheless, we emphasize that our experiments can show the feasibility of FADE in providing an additional level of security protection for today's cloud storage.

We note that the performance overhead of FADE becomes less significant when the size of the actual file content increases (e.g. on the order of megabytes or even bigger). Thus, FADE is more suitable for enterprises that need to archive large files with a substantial amount of data. On the other hand, individuals may generally manipulate small files on the order of kilobytes. In this case, we may consider the techniques of associate the same policy metadata with multiple files (see Section 5) to reduce the overhead of FADE.

\section{Discussion}

In this section, we discuss several design limitations that we do not address in this paper. We suggest possible enhancements that we can make to FADE.

Adding an Additional Layer of Encryption. In our current design of FADE, if the key manager colludes with the storage cloud, then the storage cloud can decrypt the files of data owner. To prevent this from happening, one solution is to add an additional layer of encryption in the data owner. The idea is that the data owner first encrypts a file with a long-term secret key, and then encrypts the encrypted file with the data key. In this way, even if the key manager colludes with the storage cloud, the files of the data owner remain encrypted.

Multiple Files with the Same Policy Metadata. In our current implementation, the operations of FADE are on a per-file basis, such that each data file has one corresponding policy metadata file (see Section 3). To reduce the metadata overhead of FADE, we can associate a batch of multiple data files (e.g., files under the same directory) with the same policy metadata and the same set of cryptographic keys (including the data key and the control keys of policies). The advantage of the batch-based approach is that we can use one single policy metadata for multiple data files. Thus, if the data files are of small size, then the batch-based approach can reduce the storage overhead due to the policy metadata.

It is possible to add a new data file into the batch of files that are currently associated with the same policy metadata. To achieve this, the data owner first downloads the policy metadata from the storage cloud and recovers the data key. Then it uses the same data key to encrypt the new file. Note that the content 
of the policy metadata remains unchanged. Also, the data key can be cached in the data owner's volatile storage so as to include new files into the batch later.

Reliability of the key manager. This work assumes several reliability features of the key manager (see Section 2.4), including: (i) implementation of the quorum scheme that improves the robustness of key management, (ii) removal of keys of revoked policies, and (iii) secure and reliable storage of keys of active policies that are not yet revoked. We plan to address these issues in future work.

\section{Related Work}

In Section 2.1, we discuss time-based deletion in $[5,14]$, which we generalize into policy-based deletion. In this section, we review other related work on protecting outsourced data storage.

Cryptographic protection on outsourced data storage has been considered (see survey in [8]). For example, Wang et al. [23] propose secure outsourced data access mechanisms that support changes in user access rights and outsourced data. Ateniese et al. [4] and Wang et al. [22] propose an auditing system that verifies the integrity of outsourced data. However, all the above systems require new protocol support on the cloud infrastructure, and such additional functionalities may make deployment more challenging.

Security solutions that are compatible with existing public cloud storage services have been proposed. Yun et al. [24] propose a cryptographic file system that provides privacy and integrity guarantees for outsourced data using a universalhash based MAC tree. They prototype a system that can interact with an untrusted storage server via a modified file system. JungleDisk [7] and Cumulus [21] are proposed to protect the privacy of outsourced data, and their implementation use Amazon S3 [2] as the storage backend. Specifically, Cumulus focuses on making effective use of storage space while providing essential encryption on outsourced data. The above systems mainly put the protocol functionalities on the client side, and the cloud storage providers merely provide the storage space.

The concept of attributed-based encryption (ABE) is first introduced in [17], in which attributes are associated with encrypted data. Goyal et al. [6] extend the idea to key-policy ABE, in which attributes are associated with private keys, and encrypted data can be decrypted only when a threshold of attributes are satisfied. Pirretti et al. [16] implement ABE and conduct empirical studies. Nair et al. [12] consider a similar idea of ABE, and they seek to enforce a finegrained access control of files based on identity-based public key cryptography. Perlman et al. [15] also address the Boolean combinations of policies, but they focus on digital rights management rather than file assured deletion and their operations of cryptographic keys are different from our work because of the different frameworks. As argued in Section 2.2, ABE and our work have different design objectives and hence different key management mechanisms. 


\section{Conclusions}

We propose a cloud storage system called FADE, which aims to provide assured deletion for files that are hosted by today's cloud storage services. We present the design of policy-based file assured deletion, in which files are assuredly deleted and made unrecoverable by anyone when their associated file access policies are revoked. We present the essential operations on cryptographic keys so as to achieve policy-based file assured deletion. We implement a prototype of FADE to demonstrate its practicality, and empirically study its performance overhead when it works with Amazon S3. Our experimental results provide insights into the performance-security trade-off when FADE is deployed in practice.

\section{Acknowledgment}

The work of Patrick P. C. Lee was supported by project \#MMT-p1-10 of the Shun Hing Institute of Advanced Engineering, The Chinese University of Hong Kong.

\section{References}

1. Amazon. SmugMug Case Study: Amazon Web Services. http://aws.amazon.com/ solutions/case-studies/smugmug/, 2006.

2. Amazon Simple Storage Service (Amazon S3). http://aws.amazon.com/s3/.

3. M. Armbrust, A. Fox, R. Griffith, A. D. Joseph, R. H. Katz, A. Konwinski, G. Lee, D. A. Patterson, A. Rabkin, I. Stoica, and M. Zaharia. Above the Clouds: A Berkeley View of Cloud Computing. Technical Report UCB/EECS-2009-28, EECS Department, University of California, Berkeley, Feb 2009.

4. G. Ateniese, R. D. Pietro, L. V. Mancini, and G. Tsudik. Scalable and Efficient Provable Data Possession. In Proc. of SecureComm, 2008.

5. R. Geambasu, T. Kohno, A. Levy, and H. M. Levy. Vanish: Increasing Data Privacy with Self-Destructing Data. In Proc. of USENIX Security Symposium, Aug 2009.

6. V. Goyal, O. Pandey, A. Sahai, and B. Waters. Attribute-Based Encryption for Fine-Grained Access Control of Encrypted Data. In Proc. of ACM CCS, 2006.

7. JungleDisk. http://www.jungledisk. com/.

8. S. Kamara and K. Lauter. Cryptographic Cloud Storage. In Proc. of Financial Cryptography: Workshop on Real-Life Cryptographic Protocols and Standardization, 2010.

9. LibAWS++. http://aws.28msec.com/.

10. A. J. Menezes, P. C. van Oorschot, and S. A. Vanstone. Handbook of Applied Cryptography. CRC Press, Oct 1996.

11. MyAsiaCloud. http://www.myasiacloud.com/.

12. S. Nair, M. T. Dashti, B. Crispo, and A. S. Tanenbaum. A Hybrid PKI-IBC Based Ephemerizer System. IFIP International Federation for Information Processing, 232:241-252, 2007.

13. OpenSSL. http://www.openssl.org/.

14. R. Perlman. File System Design with Assured Delete. In ISOC NDSS, 2007. 
15. R. Perlman, C. Kaufman, and R. Perlner. Privacy-Preserving DRM. In IDtrust, 2010.

16. M. Pirretti, P. Traynor, P. McDaniel, and B. Waters. Secure Attribute-Based Systems. In $A C M C C S, 2006$.

17. A. Sahai and B. Waters. Fuzzy Identity-Based Encryption. In EUROCRYPT, 2005.

18. A. Shamir. How to Share a Secret. CACM, 22(11):612-613, Nov 1979.

19. SmugMug. http://www. smugmug. com/.

20. W. Stallings. Cryptography and Network Security. Prentice Hall, 2006.

21. M. Vrable, S. Savage, and G. M. Voelker. Cumulus: Filesystem backup to the cloud. ACM Trans. on Storage (ToS), 5(4), Dec 2009.

22. C. Wang, Q. Wang, K. Ren, and W. Lou. Privacy-preserving public auditing for storage security in cloud computing. In Proc. of IEEE INFOCOM, Mar 2010.

23. W. Wang, Z. Li, R. Owens, and B. Bhargava. Secure and Efficient Access to Outsourced Data. In ACM Cloud Computing Security Workshop (CCSW), Nov 2009.

24. A. Yun, C. Shi, and Y. Kim. On Protecting Integrity and Confidentiality of Cryptographic File System for Outsourced Storage. In ACM Cloud Computing Security Workshop (CCSW), Nov 2009. 